\title{
Characterization of CdSe nanocrystals coated with amphiphiles. A capillary electrophoresis study
}

\author{
Slawomir Oszwaldowski • \\ Katarzyna Zawistowska-Gibula $\cdot$ Kenneth P. Roberts
}

Received: 18 July 2011 / Accepted: 13 October 2011 / Published online: 4 November 2011

(C) The Author(s) 2011. This article is published with open access at Springerlink.com

\begin{abstract}
We have synthesized CdSe nanocrystals (NCs) possessing a trioctylphosphine surface passivation layer and modified with amphiphilic molecules to form a surface bilayer. The NCs covered with single amphiphiles are not stable in aqueous solution, but a mixed amphiphilic system is shown to provide stability in solution over several months. The solutions of the modified NCs were characterized by UV-Vis absorbance, photoluminescence, and transmission electron microscopy. An electrophoretic study revealed two operational modes. The first relies on the enrichment of NCs using a micellar plug as a tool. The accumulation of NCs at the plug-electrolyte buffer interface results in a sharp peak. By controlling the electrophoretic conditions, nanocrystals were forced to exit a micellar plug into an electrolyte buffer. We conclude that a system consisting of modified nanocrystals and a micellar plug can act as a mixed pseudomicellar system, where modified nanocrystals play the role of pseudomicelles.
\end{abstract}

Electronic supplementary material The online version of this article (doi:10.1007/s00604-011-0727-8) contains supplementary material, which is available to authorized users.

S. Oszwałdowski $(\bowtie) \cdot K$. Zawistowska-Gibuła

Faculty of Chemistry, Department of Analytical Chemistry,

Warsaw University of Technology,

ul. Noakowskiego 3,

00-664 Warsaw, Poland

e-mail: slaosw@ch.pw.edu.pl

K. P. Roberts

Department of Chemistry and Biochemistry,

The University of Tulsa,

Tulsa, OK 74104, USA
Keywords Capillary electrophoresis $\cdot$ Separation . Nanocrystals $\cdot$ CdSe $\cdot$ Surfactants $\cdot$ Focusing $\cdot$ Mixed micelles

\section{Introduction}

Nanocrystals (NCs) possess unique electrooptic properties suitable for a variety of applications ranging from biological sensing to photovoltaic. Recent progress in the development of functional, versatile metallic and semiconducting NCs for use in workable devices has led to the need for methods for monitoring NCs properties, such as size distribution, surface charge, and reaction efficiencies of various surface functionalization strategies [1]. Instrumental tools for determining nanocrystals size and size distribution have been well established for UV-Vis spectrophotometry, photoluminescence, electron/probe microscopy, dynamic light (DLS) and X-ray scattering [2, 3]. However, elucidation of surface properties, particularly in solution, is less well developed. For example, modification of a crystal surface to provide water solubility by exchanging hydrophobic surface ligands with hydrophilic ligands or formation of a lipid bilayer with amphiphiles can drastically affect nanocrystals properties such as hydrodynamic radii, zeta potential, NC diffusion rates, cytotoxicity, luminescence quantum efficiency, and longevity. However, techniques such as TEM (transmission electron microscopy) and $\mathrm{X}$-ray scattering can only probe the nanocrystal inorganic core and not the surface coating [4]. As well, even when UV-Vis and DLS have been applied to estimate properties of NCs, such as hydrodynamic radius and solution stability [4], these techniques typically require large sample concentrations and are often at the lower edge of the size range capabilities of most DLS instruments. 
Capillary electrophoresis (CE) is uniquely suited, with its open tubular design, for characterization of NCs and their reaction products in solution due to the high separation efficiency, water-based separation media, as well as intrinsic migration behavior of nanostructures in an applied electric field [reviews: 5-9].

It is generally accepted that electrophoretic migration of nanoparticles is much more complex phenomenon than migration of a typical solute. For a charged spherical particle dispersed in a electrolyte the following forces have to be considered: (i) the electrostatic force exerted by the applied electric field, (ii) Stokes friction, (iii) electrophoretic retardation, and (iv) relaxation effects $[5,10]$. Depending on particle size (speaking precisely, the value of $\kappa a$-the ratio of the hydrodynamic radius of the particle ( $a$ ) to the electrical double-layer thickness $\left(\kappa^{-1}\right)$ ), Helmholtz-Smoluchowski, Hückel-Onsager or Henry's equation, respectively, should be used for characterization of the particles migration [11]. For particles at the nanoscale, the Henry's equation seems to be the most appropriate, however with the limitation that $\zeta$-potential should be $<25-50 \mathrm{mV}$ [11]. Many studies have substantiated that nanoparticles migration velocity is reliant on zeta potential and particle size $(\zeta, a)[5,6,10]$.

In order to overcome limitations enforced by those effects some non-conventional modes of capillary electrophoresis were recently tested. Thus, the successful separation of CdTe nanocrystals according to size with the size span $2.6 \mathrm{~nm}$ was recently proposed with the use of capillary gel electrophoresis with polymer as sieving medium [12]. The method was recently extended toward $\mathrm{CdSe} / \mathrm{ZnS} \mathrm{NCs}$ [13]. Next, the reversed electrode polarity stacking mode of $\mathrm{CE}$ with the use of anionic surfactant was examined for the enrichment of $\mathrm{Au}$ nanoparticles and an increase in the detection of particles, up to $10^{3}$ fold was reported [14-16]. Also, analytical capillary isotachophoresis was tested for the characterization (effective charge, $\zeta$ potential) of semiconductor nanoparticles [17].

The present work is an extension of works recently released [18-20], where amphiphile coated CdSe NCs were used to the characterization of electrophoretic phenomena for particles at nano scale. It was proven that coating of nanostructures with amphiphilic molecules can be a convenient way in order to solubilize them in aqueous solutions, thereby introducing a surface charge at $\mathrm{NC}$ /water interface with consequent electrostatic double layer sufficient for the particles migration under aqueous electrophoresis conditions. For this anionic, cationic as well as non-ionic amphiphiles can be used, which assure appropriate electrostatic character of a coated NCs. Coated in this way NCs can be regarded as an equivalent of NCs with voluminous ligands, which can be either a model for NCs with bulky biological ligands (charged or uncharged) or as a convenient test particle for evaluating novel analytical procedures for bio- functionalized NCs. This part of the work was supported by molecular modeling at $a b$ inito DFT level, to support the experimental data in relation to ligands exchange or $\mathrm{CdSe}$ NCs coating with amphiphilic molecules. This tool was previously applied in work [20] for estimating interactions surface of crystal CdSe and ligands.

As the consequence of coating phenomena a novel electrophoretic technique was introduced recently, where a micellar plug was applied as a tool for the preconcentration/ separation of amphiphile coated nanocrystals $[19,20]$. This is a direct consequence of phenomena observed in work [18], where an interaction of amphiphile coated CdSe NCs with regular micelles was found to be higher than NCs affinity to aqueous phase. Based on recently released works [18-20], it can be concluded that the main features of the novel system were as follows. The NCs coated with voluminous ligands (amphiphile, DNA) can be regarded as a micelle-like object (a pseudomicelle), able to form a mixed pseudomicellar system with regular micelles. Because of this, the NCs can migrate within a micellar plug, which allows NCs to accumulate at micellar/ micelle-free boundary, in the form of a sharp peak (focusing). This is the enrichment phenomenon with the enrichment efficiency $\sim 20$ (SEF $_{\text {height }}$ sensitivity enhancement factor) [19]. Modifying the electrophoretic conditions, the NCs release from a micellar plug to background electrolyte (BGE), in controllable fashion, was available. Formally, this is demixing of mixed pseudomicellar phase, the phenomenon of highly interest to the colloidal science. Note that the scheme outlined above is a new tool in the separation science that particles able to form a pseudomicellar system can be separated from these unable to create such a system $[19,20]$.

In this context, it is worth noting, that the previous studies indicate that several hydrophobic nanostructures can be made water soluble using amphiphilic molecules. These include nanocrystals [21-26], single-walled nanotubes [2729] and fullerenes [30]. In each case, an ionic head group of an amphiphile introduces a surface charge on a nanostructure with consequent electrostatic double-layer sufficient for the separation under aqueous electrophoretic conditions. These solubilization techniques have led to additional encapsulation procedures [3], separation methods using centrifugation [31], and capillary electrophoresis separations [18-20, 30, 32, 33]. In work [21], a shift in color profile of $\mathrm{Au}$ nanoparticles coated with molecules (e.g. surfactants) was related to particles electrophoretic mobility, which can a sign of complexity in the nanoparticles/CE system. Issued last works [34, 35], dealing with nanoparticles dispersed in solution of reversed micelles, show that effects: nanoparticles separation, enrichment for charged nanoparticles and coagulation, can be explained by DLVO theory (Deryaguin-Landau-Verwey-Overbeek). 


\section{Experimental}

\section{Reagents}

All chemicals and reagents used herein were of analytical grade. For synthesis of the TOP-coated CdSe nanocrystals, cadmium oxide ( $\sim 1$ micron, $99.5 \%)$, selenium powder (100 mesh, 99,999\%), trioctylphosphine (TOP, 90\%) from Sigma-Aldrich (St. Louis, USA, http://www.sigmaaldrich. com) and 1-octadecene (ODE) from Acros Organics (Morris Plains, USA, http://www.acros.be/) were used. Aqueous amphiphile solutions were prepared from SDS (sodium dodecyl sulfate), DOSS (dioctyl sulfosuccinate sodium salt), CTAB (cetyltrimethylammonium bromide), Triton N-101 (polyoxyethylene nonylcyclohexyl ether), Triton X-100 (polyoxyethylene (10) isooctylphenyl ether), Brij 30 (tetraethylene glycol dodecyl ether), Igepal CO-210 (polyoxyethylene (2) nonylphenyl ether, average $\mathrm{M}_{n}=294$ ), PVP (polyvinylpyrrolidone) average $\mathrm{M}_{\mathrm{W}}$ 1,300,000, sodium cholate, CHAPS \{3-[(3-cholamidopropyl)-dimethylammonio]-1-propanesulfonate $\}$ from Sigma-Aldrich, 1-propanesulfonic acid sodium salt, 1-pentanesulfonic sodium acid sodium salt, 1-dodecanesulfonic acid sodium salt form (AppliChem, Darmstadt, Germany, http://www. applichem.com) and sodium oleate (OA) or sodium laurate (LA) from Spectrum Chemicals (New Brunswick, USA). Structures of the compounds used for modification of CdSe NCs are presented in Fig. S1 (Electronic Support Section). Electrolyte buffers based on sodium tetraborate (Sigma-Aldrich) were prepared in the range 5-40 mM. For modification of electrolyte buffer methanol $(\mathrm{MeOH})$ and acetonitrile (ACN) were used (Fisher Scientific, New Jersey, USA, http://www.fishersci.com).

\section{Instrumentation}

The TEM unit was used in this work: JEOL JEM-2100 Scanning Transmission Electron Microscope (STEM) (JEOL Tokyo, Japan, http://www.jeol.com/) was operated at $200 \mathrm{kV}$. Images were recorded with a CCD camera controlled by AMT Image Capture Engine. 5\% deviation in size measurement was observed from the instrument. A Formvar coated copper grid, was used. Conductometry: Elmetron CPC-551 meter with Eurosensor EPS-22 N electrode (Zabrze, Poland, http://elmetron.com.pl/).

All electropherograms were obtained using a P/ACE MDQ CE system from Beckman-Coulter Instruments (Fullerton, CA, https://www.beckmancoulter.com/). System operation and data collection were controlled using Karat 5.0 software supplied by Beckman-Coulter Instruments. CE experiments were performed with the use of $75 \mu \mathrm{m}$ i.d. $\times 70 \mathrm{~cm}$ long fused-silica capillaries (Polymicro Technologies, Phoenix, AZ, http://www.polymicro.com/). The length from injector to detector was $60 \mathrm{~cm}$. The capillary was conditioned daily by rinsing for $15 \mathrm{~min}$ with $0.1 \mathrm{M} \mathrm{NaOH}$ followed by $3 \mathrm{~min}$ with water and 15 min with running buffer. Injection was performed hydrodynamically by applying typically $50 \mathrm{mbar}$ for $6 \mathrm{~s}$. Between runs, the capillary was rinsed with $0.1 \mathrm{M}$ $\mathrm{NaOH}$ for $5 \mathrm{~min}$ and running buffer for $5 \mathrm{~min}$. The separation capillary was temperature controlled at $25^{\circ} \mathrm{C}$ by liquid cooling. For experiments (Figs. 5, 6 and S10) the length of the sample plug in capillary was established. Available computers programs were unsuitable for this purpose as injection concerns two phases. This was done manually by measuring a plug length injected into capillary under appropriate conditions (time, pressure).

\section{Procedures}

\section{Preparation of TOP-coated CdSe nanocrystals}

The hydrophobic TOP-coated CdSe nanocrystals were prepared from cadmium oxide and elementary selenium using referenced procedures [36]. Briefly, the selenium precursor was prepared by combining elementary selenium and TOP at room temperature. The cadmium precursor was prepared from cadmium oxide, ODE, and oleic acid at $250{ }^{\circ} \mathrm{C}$. Prepared solutions were mixed at different temperatures for the appropriate time period to obtain the desired size of CdSe nanocrystals. The resulting CdSe/TOP nanocrystals were purified by adding a large volume of acetone followed by adding methanol until a slightly turbid solution was obtained. The mixture was left to stand overnight. Larger NCs precipitated after the mixture stood overnight, but smaller NCs required centrifugation (3,000 rpm, $15 \mathrm{~min})$. The pure nanocrystals were washed with methanol and then dissolved in either toluene or hexane for extended storage or in chloroform for the preparation of amphiphile-coated NCs.

\section{TEM sizing of core CdSe QDs}

The size of CdSe core structures $\left(2.8 \mathrm{~nm}\right.$; UV-VIS $\lambda_{\mathrm{abs}}=$ $537 \mathrm{~nm}$ ) was measured using the JEOL JEM-2100 Scanning Transmission Electron Microscope (STEM) (Oklahoma State University). One drop of a dilute sample of $\mathrm{CdSe} / \mathrm{TOP} \mathrm{NCs}$ in chloroform or $\mathrm{CdSe} / \mathrm{TOP} / \mathrm{N}-101$ solution prepared in $10 \mathrm{wt} . \% \mathrm{~N}-101$ was placed onto a Formvar coated copper grid, allowed to settle for $20 \mathrm{~s}$, and wicked away using an absorbent tissue. Size analysis was performed on digital images captured with an ImageJ V. $1.34 \mathrm{~s}$ program.

\section{Nanocrystal surface modification}

The samples of modified CdSe/TOP NCs were prepared by adding $200 \mu \mathrm{l}$ chloroform solution of $\mathrm{CdSe} / \mathrm{TOP}$ 
$\left(1.5 \times 10^{-5}-3 \times 10^{-6} \mathrm{M}\right.$, depending on $\mathrm{NC}$ size $)$ to the mixture of non-ionic surfactant (TX-100) and active amphiphile: ionic surfactants, non-ionic surfactants or polymer (PVP example). Typically the surfactant mixture was $0.5 \mathrm{~g}$ TX-100 (10\%; w:w, water solution) and $27-35 \mathrm{mg}$ of active amphiphile. Only in the case of N-101, the samples were prepared with the use of $10 \% \mathrm{w}: \mathrm{w}$ water solution of $\mathrm{N}-$ 101. The mixture was left to stand overnight at room temperature, in the dark area, with stirring with a magnetic stir bar in order to evaporate chloroform and coating. Details of the composition of a surfactant solution appear in the figure caption, which illustrates an appropriate effect. Samples before CE experiment were centrifuged $(15.000 \mathrm{rpm} / 10 \mathrm{~min})$ in order to remove aggregates.

\section{Capillary electrophoretic separation}

Sodium tetraborate (5-40 mM) electrolyte buffers were used for capillary electrophoresis. The electrolyte buffers (typically $5 \mathrm{mM}$ or $10 \mathrm{mM}$ sodium tetraborate) were modified by adding up to 40 vol. $\%$ methanol $(\mathrm{MeOH})$ or acetonitrile $(\mathrm{ACN})$. Details of the composition of an electrolyte buffer appear in the figure caption, which illustrates an appropriate effect. Sample solutions were injected into the capillary from the anode side. Separations were done by applying voltages in the range of $3-25 \mathrm{kV}$. It is recommended to run the mixture (a pair of ionic/non-ionic amphiphiles) 2-3 times before nanocrystals separation. The peak parameters (peak area, height, width, and spectrum) were obtained with Karat 5.0 software. The number of theoretical plates was calculated according to ref. [37, page 870$]$ as $N=5.54\left[\left(\mathrm{t}_{\mathrm{i}} /\left(\text { peak area } / \mathrm{i}_{\mathrm{i}} / \mathrm{peak}_{\mathrm{high}}\right)\right)\right]^{2}$.

\section{Results and discussion}

\section{Synthesis and characterization of CdSe/TOP NCs}

CdSe/TOP NCs with different size from 2.1 to $3.7 \mathrm{~nm}$ in diameter were synthesized and purified using reported procedures $[2,36]$. Determinations of a crystal size were accomplished by well-established UV-Vis and TEM methods [2, 36, 38, 39]. In brief, initial NCs size and concentration determinations were based on the position and intensity of the first exciton band in the UV-Vis spectra. The extinction coefficient and spectral position of the 1S3/2-1Se excitonic absorption band for CdSe nanocrystals, as a function of size, are determined from the empirical functions given in a recent work of $\mathrm{Yu}$ et al. [2] (valid in a size range between 1 and $8 \mathrm{~nm}$ ). Confirmation of the crystal sizes was accomplished using high-resolution TEM (Fig. S2, supporting materials). As previously observed, there was a slight negative $(\sim 0.5 \mathrm{~nm})$ bias in the UV-Vis determinations relative to TEM, in agreement with ref. [36].
Modification of surface of CdSe/TOP NCs

Hydrophobic CdSe/TOP NCs were phase transferred into aqueous media by forming a reverse-lipid bilayer on NC surface, using an amphiphilic molecules, i.e., $\mathrm{CdSe} / \mathrm{TOP} \rightarrow \mathrm{CdSe} / \mathrm{TOP} / /$ amphiphile. This was facilitated using non-ionic, ionic, and polymeric amphiphilic molecules shown in Figure S1. It was found that among these amphiphiles the most stable aqueous solution for CdSe NCs coated with amphiphiles, was obtained using R-COONa derivatives (LA, OA) or non-ionic surfactant Triton N-101 ( $\geq 1$ week). Amphiphiles (R-SO ${ }_{3} \mathrm{Na}$ : DOSS, SDS) provide stable dispersion for $\mathrm{CdSe} / \mathrm{TOP} \mathrm{NCs}$, for more than just a few hours, where larger NCs $(>3 \mathrm{~nm})$ were the least stable. Many other single amphiphile coatings did not allow for adequate NCs dispersion in aqueous medium or quickly aggregates. Note that an appropriate concentration range of an amphiphile is necessary for effective dispersion of $\mathrm{CdSe} /$ TOP NCs and maintaining the highest as possible PL of NCs. In this situation (Fig. 1), the NCs aggregation occurs at lower concentration of an amphiphile, due to lack of effective coating, whereas the limit of the highest amphiphile concentration relies on the its intrinsic solubility in water.

Additional experiments revealed, that the long term stability ( $>4$ months) of amphiphiles coated CdSe/TOP $\mathrm{NCs}$ was obtained using a pair of ionic/non-ionic amphiphiles for NCs coating. Examples of this system are shown for the visual inspection (Fig. S3, supporting materials). In this case, the NCs dispersed in solution of a pair of ionic/non-ionic amphiphiles were coated with ionic amphiphiles. This is based on the observation that both native $\mathrm{CdSe} / \mathrm{TOP} \mathrm{NCs}$ dissolved in a organic solvent (hexane, chloroform) and this coated only with the nonionic TX-100 amphiphile (CdSe/TOP//TX-100) have high level of normalized photoluminescence (PL per 0.1 absorbance unit, Fig. 2b). This was significantly reduced due to NCs coating with a pair of ionic/non-ionic amphiphiles (Fig. 2b), despite the highest NCs solubilization obtained in this situation (Fig. 2a). Both parameters (AU, PL) were summarized in Fig. 2c, d for representative anionic, non-ionic

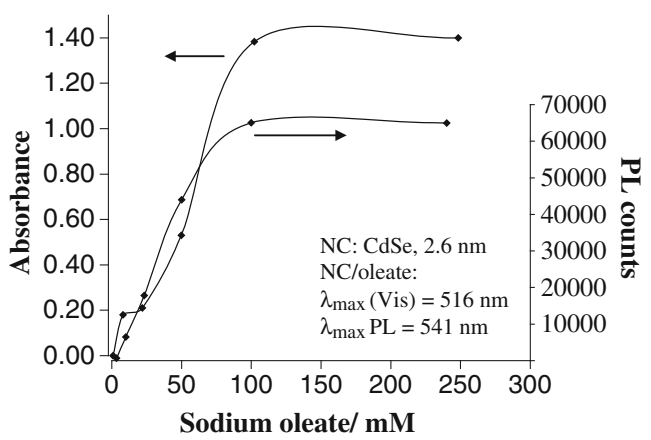

Fig. 1 Dispersion of CdSe NCs in aqueous solution of sodium oleate 
a

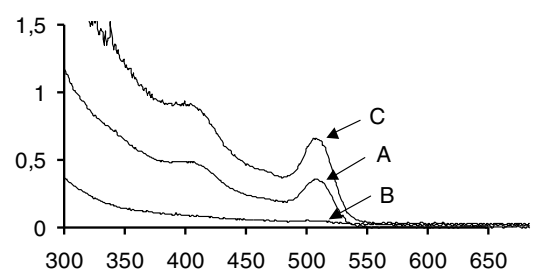

b

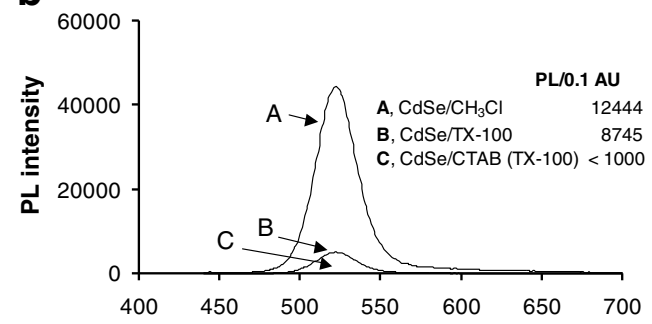

Wavelength/ nm

c

d
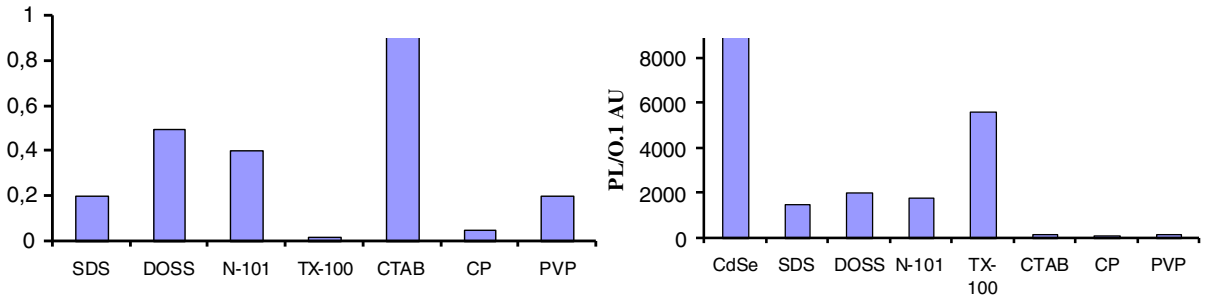

surfactant

Fig. 2 Upper (a, b). Spectra for CdSe NCs due to solubilization of CdSe/TOP NCs $(2.4 \mathrm{~nm}$, first excition band, $\lambda=508 \mathrm{~nm})$ using a nonionic or a pair of ionic/non-ionic surfactants. a, UV-Vis spectra, b, photoluminescence spectra (PL). Samples of CdSe NCs: A, in chloroform, B, dissolved in TX-100 and C, in mixture of TX-100/ CTAB. As an insert in (b) is comparison of the normalized emission efficacy (PL per $0.1 \mathrm{AU}$ ) reflecting the PL effect, due to NCs solubilization in these surfactants. Below $(\mathbf{c}, \mathbf{d})$. Coating and emission efficacy due to solubilization of CdSe/TOP NC in selected pairs of amphiphilic surfactants. Data summarized for CdSe NCs sizes $=2.7-$ $3.3 \mathrm{~nm}$. Figures: c, coating efficacy in the form of absorbance of the NCs solution (first exciton band of NC in UV-Vis); $\mathbf{d}$, the normalized

and cationic surfactants, examined in the present work, with the same conclusion.

These observations were supported by theoretical data for the CdSe crystal and selected ligands in terms of ligands binding to the crystal surface [20]. For this the theoretical tool - molecular modeling at DFT level was applied (Electronic Support Section, Figs. S13, S14, S15, S16 and S17). Both results (experimental and theoretical) lead to the one general conclusion that there are, at least, two coating mechanisms for CdSe NCs using amphiphilic molecules. This can be explained based on a model of the passivation of InP nanocrystals by TOPO (trioctylphosphine oxide) ligands [40], shortly discussed in Electronic Support Section, that passivation relies on two phenomena: (i) crystal-ligand, and (ii) ligand-ligand interaction, both having similar importance in terms of binding energy. For CdSe/amphiphile system, analysed in the present work, it can be stated that dispersion of CdSe/TOP NCs using only $\mathrm{R}_{-} \mathrm{SO}_{3}{ }^{-}$amphiphile (SDS, DOSS) leads to less stable CdSe/ amphiphile system. This can be concluded from experimentally demonstrated short-term NCs stability, lack of a peak for CdSe/amphiphile NCs during CZE run, as well as from the theoretical model. In this case, based on the model emission efficacy (PL per $0.1 \mathrm{AU}$ ) for NCs solutions according to frame c. Abbreviations: CdSe, chloroform solution of CdSe $(50 \mu \mathrm{l}$ of $2-510^{-6} \mathrm{M}$ CdSe NCs per $500-300 \mu \mathrm{l}$ of chloroform). Coating with: only non-ionic surfactants (TX-100, N-100; 10\% w:w) (examples of coating with single amphiphile) and pairs of surfactants: $10 \%$ TX-100 (w:w) containing $100 \mathrm{mM}$ of ionic surfactants: (anionic) SDS sodium dodecyl sulphate; DOSS, dioctyl sulfosuccinate; (cationic) CTAB, cetyltrimethylammonium bromide; $\mathrm{CP}, 1$-dodecylpyridinium chloride. Sample preparation: $\left(50 \mu \mathrm{l}\right.$ of $2-510^{-6} \mathrm{~mol} \mathrm{l}^{-1} \mathrm{CdSe} / \mathrm{TOP}$ NCs per $300 \mu \mathrm{l}$ of surfactant solution). An example of CdSe/TOP being solubilized in a pair of non-ionic surfactant/polymer (TX-100/PVP; $0.5 \mathrm{~g} 10 \% \mathrm{TX}-100$ and $0.022 \mathrm{~g} \mathrm{PVP}$ ) is shown for comparison

of the surface passivation, it can be stated that surface ligand - amphiphile (TOP $\leftrightarrow$ amphiphile) interaction is the main cause for dispersing $\mathrm{CdSe} / \mathrm{TOP} \mathrm{NCs}$, whereas interaction: crystal - amphiphile, can be negligible. On the other hand, the $\mathrm{CdSe} / \mathrm{TOP} \mathrm{NCs}$ coating with only $\mathrm{R}-\mathrm{COO}^{-}$ amphiphile (LA, OA) ensures more stable CdSe/amphiphile system, as concluded from enhanced stability of NCs over time, the presence of a peak for NCs in CE, as well as theoretical model. In this case, the binding of amphiphiles, according to passivation model, is due to both interactions (crystal-amphiphile and surface ligand - amphiphile, respectively), which definitely enhance the $\mathrm{CdSe} / \mathrm{TOP} / \mathrm{R}$ $\mathrm{COO}^{-}$overall stability.

In the present work, it was stated that $\mathrm{CdSe} / \mathrm{TOP}$ $\mathrm{NCs}$ were better dispersed using a pair of amphiphilic molecules (non-ionic/ionic) than using an individual amphiphile. In this case, another effect should be considered, that nanocrystals can be incorporated into a micelle structure. According to the ref. [22] surfactants with single alkyl chain did not form a structure of pseudoaggregate (NC trapped inside micelle) and limited stability for the structure was found using surfactant with two alkyl chains (eq. DOSS). The best results in terms of 
stability of a pseudoaggregate have been reported using a mixture of surfactants (a mixture of n-poly(ethylene glycol) phosphatidylethanolamine (PEG-PE) and phosphatidylcholine (PC)), where a pseudoaggregate was found to be more stable over time, compared with this, applying the individual PEG-PE or PC micelle. In the present work, a micelle of non-ionic amphiphile (TX-100, N-101) was applied. Specifically for TX-100, the micelle radius $4.3 \mathrm{~nm}$ and even wider was reported, depending on actual micelle shape [41]. This allows build a temporary pseudoaggregate containing nanocrystal (core and surface hydrophobic ligands) trapped inside a micelle structure or covered by a layer or bilayer of amphiphiles. In this situation, a direct contact between CdSe/TOP NCs and aqueous phase can be restricted and possible follow-up reactions, under the well-known term-microreactor, are available. This is supported by literature, where surfactant micelles or microemulsion droplets [24, 25, 42] were applied to transfer NCs between phases, in order to build a protective layer around a $\mathrm{CdSe} / \mathrm{ZnS}$ nanocrystal (case of silica coating). This effect can facilitate attachment of selected amphiphiles to a surface of CdSe crystal, which acting alone (single amphiphile) can slowly/incomplete substitute or coat native surface ligands (TOP, TOPO), promoting nanocrystals aggregation in aqueous conditions, as mentioned above.

To summarize, coating with a pair of ionic/non-ionic amphiphiles, can be an effective tool to disperse the nanocrystals in aqueous media, even using amphiphilic molecules with low binding energy between a crystal and an amphiphile or even in this situation, where amphiphiles can passivate only selected faces of a nanocrystal, as concluded from the theoretical model (Electronic Support Section).

\section{Migration behavior of amphiphile coated CdSe NCs}

Based on CZE, the preliminary experiments using CdSe NCs coated with a particular ionic amphiphile (SDS, DOSS, LA or OA) confirm conclusions given in the previous section. Therefore a peak for these NCs coated with $\mathrm{R}^{-\mathrm{COO}^{-}}$amphiphiles (OA, LA) was obtained. Contrary to this, no peak for these NCs was seen, applying R$\mathrm{SO}_{3}{ }^{-}$amphiphiles (DOSS, SDS) for coating. In the latter case, the peak absence is due to amphiphiles come off from the CdSe crystal as these amphiphiles have been defined as a weakly bound to facets of the CdSe crystal.

An interesting capillary electrophoretic phenomena have been reported recently $[19,20]$, when a non-ionic amphiphile was applied to the sample containing nanocrystals coated with ionic amphiphiles. In this situation, using CZE or MEKC, the peak of NCs was visualized, irrespective of applied an ionic amphiphile used for NCs coating (weakly or strongly bound to CdSe NCs). An example of this, namely NCs dispersed in TX-100/DOSS was included to electronic support section (Fig. S4, supporting materials). This example also explains the detection scheme applied in the present work, that wavelength $\lambda=245 \mathrm{~nm}$ is suitable for detecting both micellar plug and NCs simultaneously, whereas $\lambda>300 \mathrm{~nm}$ is selective for NCs only.

The aim of the present section is a deeper insight into the migration mechanism for $\mathrm{NC} /$ plug system. For this water soluble, electrically neutral CdSe/TOP//N-101 NCs (CdSe NCs dispersed in the non-ionic N-101 amphiphile) was applied due to results presented in the previous section (Fig. 2), that N-101 affords for better NCs dispersion in aqueous solution. Moreover, aqueous solution of such modified NCs was found to be stable over time and no aggregation or precipitation was seen for at least 1 week.

Further discussion will be conducted on the basis of three experiments (Fig. 3 and Figs. S5, S6, Electronic Support Section). In the first experiment (Fig. 3), to the sample containing $\mathrm{CdSe} / \mathrm{TOP} / \mathrm{N}-101 \mathrm{NCs}$ dispersed in solution of non-ionic amphiphile (N-101), the ionic amphiphile (R$\mathrm{SO}_{3} \mathrm{Na}$ ) was added followed by CZE run using sodium tetraborate BGE. The ionic amphiphile was $\mathrm{R}-\mathrm{SO}_{3} \mathrm{Na}$ with short $\left(\mathrm{C}_{3}, \mathrm{C}_{5}\right)$ or long $\left(\mathrm{C}_{12}\right)$ carbon chain, respectively. The former both do not form micelles, whereas the latest does at cmc equal to $9.8 \times 10^{-3} \mathrm{M}$ [41]. Electropherograms (frame $\mathrm{b}$ and c) show distinctive difference, when both types of R$\mathrm{SO}_{3} \mathrm{Na}$ amphiphiles were applied to a sample plug. In the first instance $\left(\mathrm{R}=\mathrm{C}_{3}, \mathrm{C}_{5}\right)$ the position of a peak of $\mathrm{CdSe} /$ TOP/N-101 was close to EOF. In the second case $\left(R=C_{12}\right)$, the shift of the peak of $\mathrm{CdSe} / \mathrm{TOP} / \mathrm{N}-101$ to the plug/BGE boundary position was seen.

From Fig. 3 it can be stated that superposition of two effects enables NCs to migrate under new (NC/plug) term. The first requirement is the presence of a plug consisting of mixed micelles and this is archived by injecting a pair of amphiphiles - a non-ionic amphiphile (e.g. TX-100, N-101) mixed with an ionic amphiphile having long hydrocarbon chain (frame c). Note, that a non-ionic amphiphile alone or mixed with an ionic amphiphiles with the short hydrocarbon chain were unable to form a phase of mixed micelles (plug), that a plug migrates near EOF position (frame a, b). This is in agreement with ref. [43], where formation of a segment of a micellar phase in between BGE was observed. In this case a length of the segment (plug) in between fixed BGE depends on the ratio non-ionic/ionic surfactant in the micellar phase. Thus, applying only a non-ionic surfactant no segment was seen and further widening of the segment is in parallel with an increase in the content of an ionic amphiphile in the phase.

The second effect is the migration of uncharged $\mathrm{CdSe} /$ TOP/N-101 NCs from the entire plug to the plug/BGE boundary. Surely, the expected result would be a peak at 

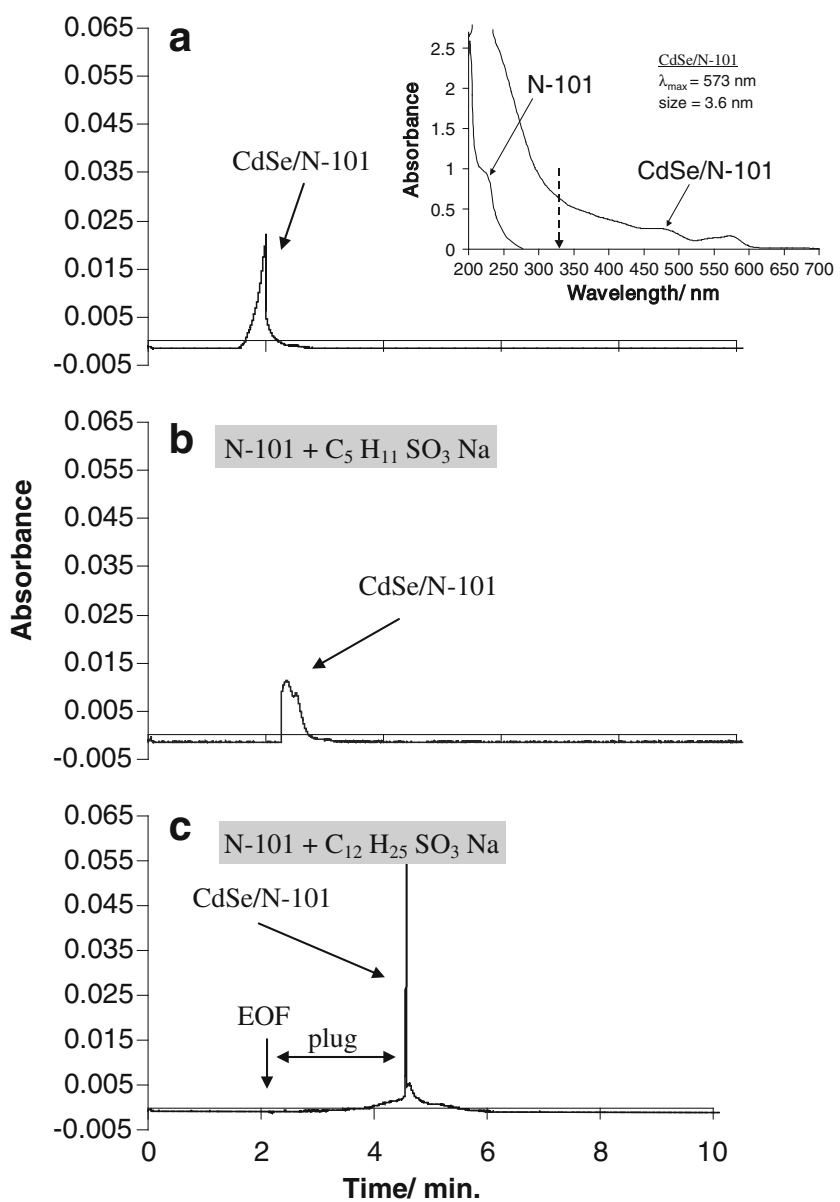

Fig. 3 Effect of a kind of an anionic amphiphile in the sample plug on the formation of a focussed peak for CdSe/TOP//N-101 NCs. Sample: $\mathrm{CdSe} / \mathrm{TOP} \mathrm{NCs}$, size $3.6 \mathrm{~nm}$, dispersed in non-ionic amphiphile $\mathrm{N}$ $101(10 \%)$. Injection: a, only sample of CdSe/TOP//N-101 NCs/10\% $\mathrm{N}-101$ (w/w); b, sample of CdSe/TOP//N-101 NCs containing N-101 $5 \%(\mathrm{w} / \mathrm{w}) / 0.28 \mathrm{M} \mathrm{C}_{5} \mathrm{H}_{11} \mathrm{SO}_{3} \mathrm{Na}$; $\mathbf{c}$, sample of $\mathrm{CdSe} / \mathrm{TOP} / \mathrm{N}-101 \mathrm{NCs}$ containing $\mathrm{N}-1015 \%(\mathrm{w} / \mathrm{w}) / 0.22 \quad \mathrm{M} \mathrm{C}_{12} \mathrm{H}_{25} \mathrm{SO}_{3} \mathrm{Na}$. Conditions: electrolyte buffer, $10 \mathrm{mM}$ sodium tetraborate; voltage, $25 \mathrm{kV}$; injection $50 \mathrm{mbar} / 0.1 \mathrm{~min}$; detection $330 \mathrm{~nm}$. Inset, frame a, shows spectra for non-ionic $\mathrm{N}-101$ and $\mathrm{CdSe} / \mathrm{TOP} / \mathrm{N}-101$, respectively. The vertical arrow shows the detection wavelength selective for $\mathrm{NCs}$, applied to $\mathrm{CE}$ experiment

EOF position for neutral NCs. Therefore, an appearance of a peak of NCs at the plug/BGE border denotes the presence of charged NCs in this situation; this despite injection of uncharged NCs. In this case, the transformation of an electrically neutral $\mathrm{NC}$ into a negatively charged $\mathrm{NC}$, because of additional coating with negatively charged $\mathrm{R}$ $\mathrm{SO}_{3}{ }^{-}$amphiphile or partial exchange of surface amphiphiles $\left(\mathrm{N}-101 \rightarrow \mathrm{R}^{-} \mathrm{SO}_{3}{ }^{-}\right)$takes place inside a plug of mixed micelles, during a run. This effect is in agreement with ref. [44], where dynamic coating of gold nanoparticles by SDS molecules during MEKC run was reported. This allows $\mathrm{NC}$ to be transformed into a micelle-like object (pseudomicelle) and a micellar type interaction between regular micelles and amphiphile coated NCs (pseudomicelles) takes place that mixed pseudomicellar phase can be formed. In this situation, migration of NCs within $\mathrm{NC} /$ plug system is controlled solely by migration phenomena available for mixed micelles, e.g. length of the plug, as confirmed later (Figs. 5, 6, S10). Note, that the effect is universal and applying a pair of amphiphiles as a plug, the accumulation of various nanostructures at plug/BGE boundary was reported recently (NC/DNA, NC/silica shell/DNA or NC/derivative of 1,10-phenathroline) [19, 20]. In this case, the transformation of a nanostructure (e.g. NC/DNA) into a micellelike object (NC/DNA//amphiphile) enables build a pseudomicellar system with regular micelles. Based on this, nanoparticles able to build a stable pseudomicellar system can be distinguished from those unable to do this by electrophoresis [19].

Next experiments, based on MEKC methods (Figs. S5, S6, supporting materials), are continuation of discussion presented in the previous works [18-20]. The aim of the Fig. S5 was to demonstrate the presence of a strong interaction between a CdSe/amphiphile and a regular micelle, which enforces the CdSe/amphiphile to migrate along with a micellar phase. In this situation, in all cases, a shift in peak position to $\mathrm{k}^{\prime}=\infty$ position for charged or uncharged NCs (e.g. from $\mathrm{k}^{\prime}=0$ to $\mathrm{k}^{`}=\infty \rightarrow$ neutral NCs) does support the idea about pseudomicellar phase discussed upper. Figure S6 depicts an impact of the concentration of a non-ionic surfactant in the sample plug and confirms the role of a non-ionic surfactant in the transformation of peak of CdSe/amphiphile NCs. In this case, the observed transformation: peak (MEKC) $\rightarrow$ peak (focused NCs) is in agreement with similar transformation using CZE method applied to CdSe NCs coated with LA, as reported in ref. [19].

\section{Focusing effects for CdSe/TOP//amphiphile NCs}

In the following sections, $\mathrm{NCs} /$ plug system, based on only charged NCs, is discussed. Moreover, only CZE mode was applied in order to avoid additional equilibria related to MEKC mechanism. Note, that NCs coating with ionic amphiphiles, which leads to formation of electrically charged NCs, was confirmed twice in the present work. In the first section it was established that a decrease in PL for CdSe NCs dispersed in a pair of amphiphiles is related to $\mathrm{NCs}$ coating with an ionic amphiphile. Another proof comes from the gel electrophoresis experiment (Fig. S7, supporting materials). By comparing migration of commer-

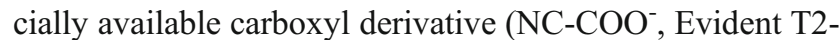
MP EviTags, carboxy functionalized) with migration of CdSe NCs coated with anionic amphiphiles, the presence of negatively charged NCs, irrespective of a way of NCs coating (by a single amphiphile, or using a pair of amphiphiles), was confirmed. Moreover, coating of NCs 
is a rapid process as the sample of $\mathrm{NC} / \mathrm{TOPO}$ mixed with the aqueous solution of an ionic amphiphile, just before GE run, shows the peak of $\mathrm{CdSe} / \mathrm{amphiphile.}$

The present section is continuation of recently released results [19, 20], dealing with focusing of NCs using NC/plug system. Several experiments, included to the present section, enable characterize the system. Based on NCs dispersed in a pair of amphiphiles, applied as the sample plug, the linear relation between peak (area, height) for focused NCs and concentration of NCs $\left(\mathrm{c}_{\mathrm{CdSe}}\right)$ (Fig. S8, supporting materials) confirms: (i) quantitative accumulation of NCs, due to transfer NCs from the entire sample plug to the plug/BGE boundary, and that (ii) the transport and accumulation concerns nanocrystals being dispersed in a plug, but not being in the form of aggregates (otherwise both migration time and peak height should be random [5]).

The transport of NCs under NC/plug system was further analysed applying various voltages. It was observed (Fig. 4, frames a-d) that a decrease in applied voltage $(20,14,10$ and $4 \mathrm{kV}$, respectively) leads to an increase in electrophoretic mobility $\left(=\mu_{\mathrm{app}}-\mu_{\mathrm{EOF}}\right)$ of negatively charged NCs. However, contrary to typical band broadening of solutes with increasing migration time, the peak of focused NCs, located in a wider micellar plug, remains sharp. Moreover, there is an apparent distinction in peak area applying low vs. high voltage (frame e). Thus, at lower voltage, a peak area of NCs reached its the highest value, which means optimal state in mutual velocities (plug vs. NCs). A decrease in a peak area at higher voltage denotes the
Fig. 4 Focusing of CdSe/TOP// SDS NCs due to migration of NCs within a micellar plug and characteristic of the peaks for CdSe coated with a pair of TX-100/SDS or TX-100/DOSS amphiphiles. Figs. a-d, visualization of focused peaks for $\mathrm{CdSe} / \mathrm{TOP} / \mathrm{SDS} \mathrm{NCs}$, core size $2.9 \mathrm{~nm}$. Voltage applied: a, $20 \mathrm{kV} ; \mathbf{b}, 14 \mathrm{kV} ; \mathbf{c}, 10 \mathrm{kV}$ and $\mathbf{d}$, $4 \mathrm{kV}$. Injection: $35 \mathrm{mbar} / 5 \mathrm{~s}$. Sample preparation: CdSe/TOP NCs dispersed in $0.5 \mathrm{~g} 10 \% \mathrm{TX}$ 100 with 0.032 g SDS. Electrolyte buffer: $5 \mathrm{mM}$ sodium tetraborate. Positions of EOF were denoted by vertical arrows. As an inset, frame $\mathbf{d}$ is DAD spectrum for $\mathrm{CdSe} / \mathrm{TOP} / / \mathrm{SDS}$ NCs obtained during a CE run. Frame e, peak area dependence on applied voltage. Electrolyte buffer: $5 \mathrm{mM}(\mathrm{A}, \mathrm{C})$ and $3 \mathrm{mM}$ (B) sodium tetraborate
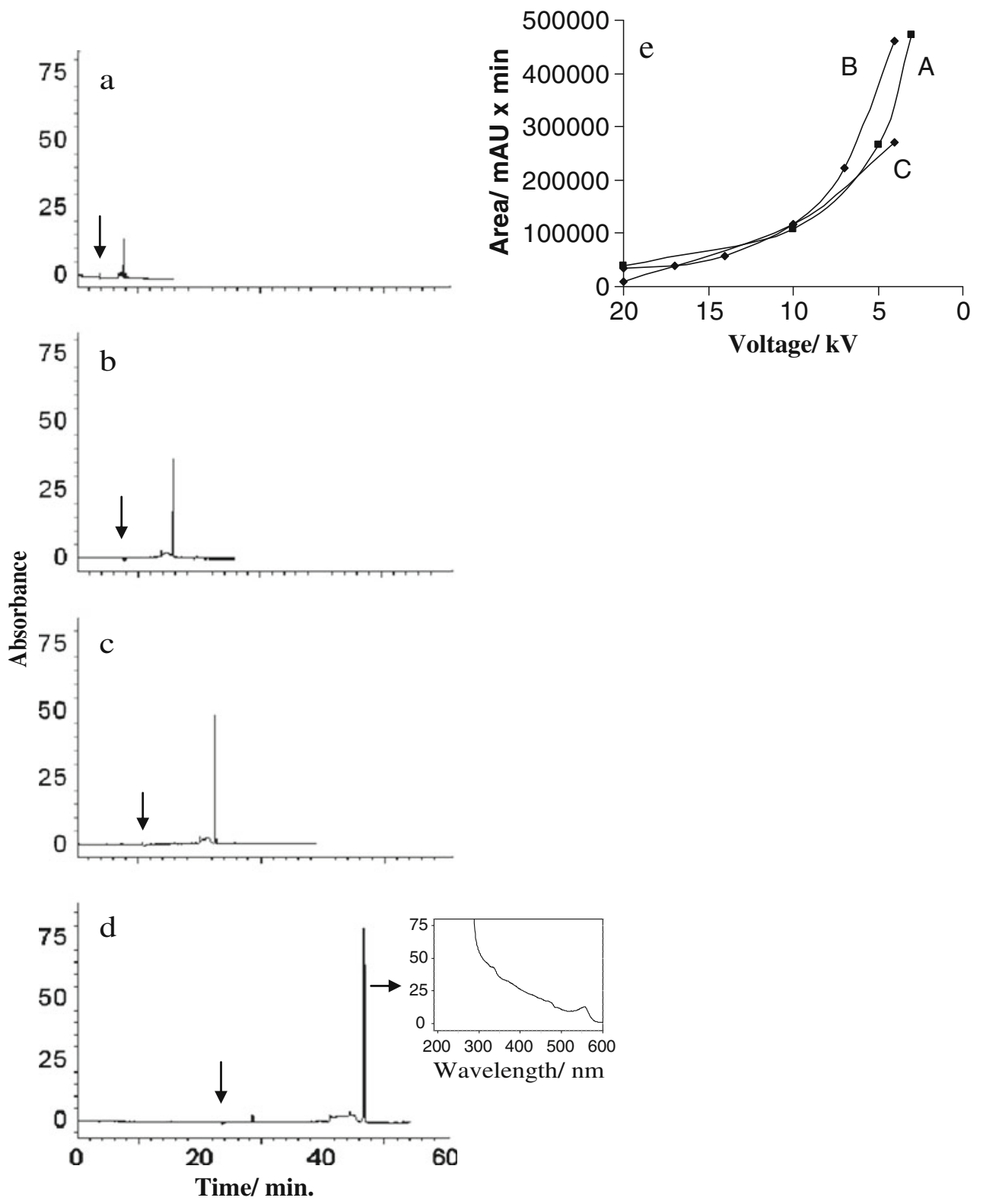
presence of side effects. This can be partial aggregation (dimers, trimers and so on) imposed by high energy field or distribution of NCs between plug and BGE, as well as NCs exit (plug $\rightarrow$ BGE).

In order to recognise the focusing effects more deeply a set of experiments were undertaken using sequence of two plugs, where a sample plug containing NCs was the first plug followed by a micellar plug free from NCs (Figs. 5, 6 and S10). In this instance, throughout all experiments (Figs. 5, 6 and S10), the sample of CdSe NCs dispersed in a pair of amphiphiles (TX-100/DOSS) (CdSe NCs, size $3.6 \mathrm{~nm}$, concentration $5.2 \times 10^{-6} \mathrm{~mol} \mathrm{l}^{-1}$ ) was the first plug, about $3.3 \mathrm{~cm}$ length. The second plug was a pair of TX100/DOSS amphiphiles. The concentration of TX-100 $(10 \% \mathrm{w}: \mathrm{w})$ in both plugs was kept constant. The experimental variables were DOSS concentration in a plug or the length of second plug, respectively.

First experiment (Fig. 5) shows an effect of a dilution of a sample plug by means of solution of TX-101 (10\% w:w). In this instance, the concentration of both (NC, DOSS) were diminished $([\mathrm{TX}-100]=$ constant $)$. A linearity between NCs peak features (area, height) and NCs concentration was seen, which confirms the similar relation discussed upper (Fig. S8, supporting materials). The meaning of the experiment is that transport phenomena for $\mathrm{NC} /$ plug system are universal for this system, this despite implementation of various configurations (one plug vs. two plugs). Also, the migration of NCs from the first plug, through the second plug, to plug/BGE boundary additionally confirms the state of NCs being dispersed in plug (no aggregation). Under this state, transport of NCs through two micellar plugs was available, under electrophoretic conditions applied.

In next experiment (Fig. S10, included to supporting materials due to many details) an impact of DOSS concentration in the second plug on the transport of NCs was analysed. For this detection, using two wavelengths allows monitor both $\mathrm{NCs}$ and plug simultaneously $(\lambda=$ $245 \mathrm{~nm})$ or NCs only $(\lambda=330 \mathrm{~nm})$, respectively. Similarly to the previous figure, the first plug was a sample plug (NCs dispersed in TX-100/DOSS; plug length $3.3 \mathrm{~cm}$ ) and the second plug was mixture of amphiphiles only (TX-100/ DOSS; plug length $6.4 \mathrm{~cm}$ ). Similarly to the previous figure, the transport of NCs throughout two plugs was the effect observed. The difference is, that a decrease in content of DOSS in the second plug, leads to: (i) shift in migration time

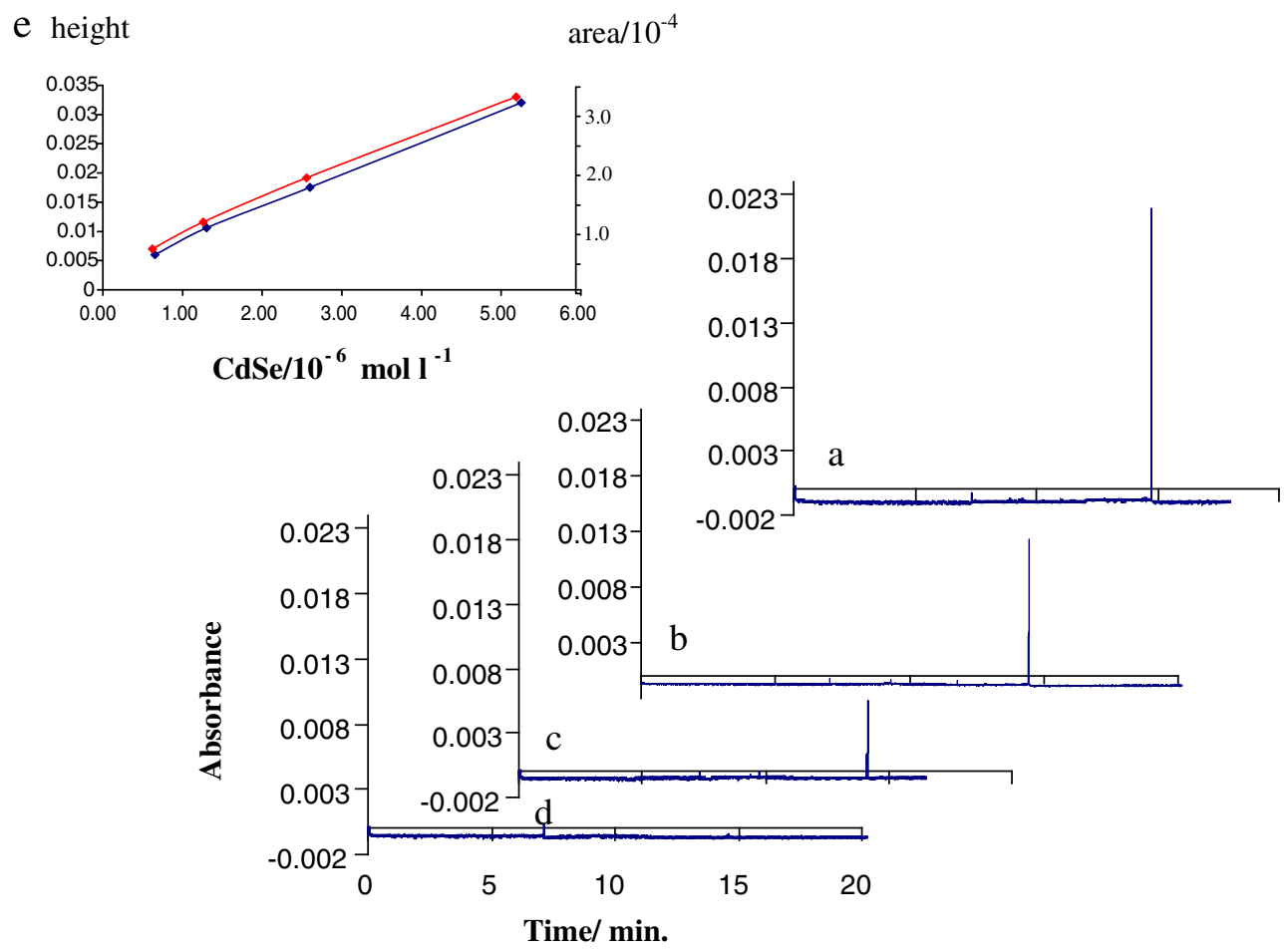

Fig. 5 Two plugs system. Impact of the concentration of an ionic amphiphile (DOSS) in the first plug on peak shape of CdSe/DOSS NCs. Frames (left side): a, two plugs system with the first plug containing $10 \%$ TX-100 (w:w), $0.148 \mathrm{~mol}^{-1}$ DOSS and $5.2 \times 10^{-6} \mathrm{~mol}^{-1} \mathrm{CdSe}$ NCs; $\mathbf{b}$, the same system, first plug: $10 \% \mathrm{TX}-100,0.074 \mathrm{~mol}^{-1} \mathrm{DOSS}$ and $2.6 \times 10^{-6} \mathrm{~mol}^{-1} \mathrm{CdSe} \mathrm{NCs}$; c, ibidem, $10 \% \mathrm{TX}-100$, $0.037 \mathrm{~mol} \mathrm{l}^{-1}$ DOSS and $1.3 \times 10^{-6} \mathrm{~mol} \mathrm{l}^{-1} \mathrm{CdSe} \mathrm{NCs}$; d, two plugs system with first plug: 10\% TX-100 and $0.148 \mathrm{~mol} \mathrm{l}^{-1}$ DOSS (blank). As the second plug, a pair of amphiphiles 10\% (w:w) TX-100, $0.148 \mathrm{~mol} \mathrm{l}^{-1}$ DOSS, were applied for (a-d). Conditions: BGE $10 \mathrm{mM}$ sodium tetraborate, applied voltage $20 \mathrm{kV}$, detection UV-vis $\lambda=330 \mathrm{~nm}$, selective for CdSe NCs (NC size, $3.6 \mathrm{~nm}$ ). Length of a plug: first $3.3 \mathrm{~cm}$, second $6.4 \mathrm{~cm}$. Right side. Frame (e), the relation between peak area (red line) or height (blue line) and $\mathrm{c}_{\mathrm{CdSe}}$ 

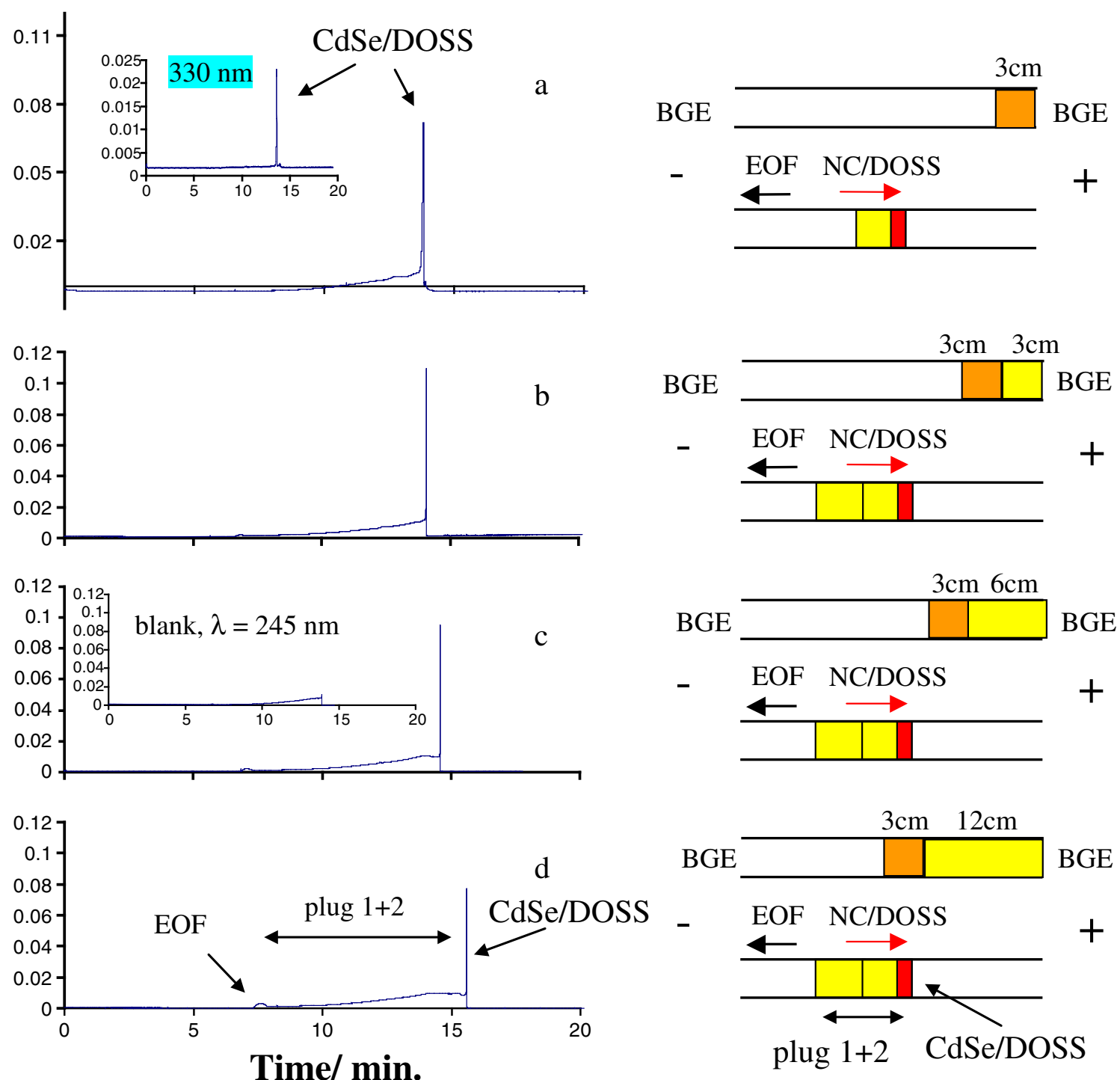

Fig. 6 Comparison of one and two plug systems, as well as impact of the length of the second plug on migration and peak shape of $\mathrm{CdSe} /$ DOSS NCs. Frames: a, one plug system (CdSe NCs, size $3.6 \mathrm{~nm}$, dispersed in TX-100/DOSS); b, two plugs system with $3.3 \mathrm{~cm}$ second plug; c, two plugs system with $6.4 \mathrm{~cm}$ second plug and $\mathbf{d}$, two plug system with $11.7 \mathrm{~cm}$ second plug. As the first plug the CdSe NCs dispersed in a pair of surfactants $(0.6 \mathrm{~g} 10 \%$ (w:w) TX-100/ $0.148 \mathrm{~mol}^{-1}$ DOSS) was applied through frames a-d, plug length $3.3 \mathrm{~cm}$. As the second plug a pair of surfactants $(0.6 \mathrm{~g} 10 \%$ (w:w) TX$100 / 0.148 \mathrm{~mol} \mathrm{l}^{-1}$ DOSS) with various lengths $(3.3,6.4$ and $11.7 \mathrm{~cm}$,

respectively) was applied. Conditions: $\mathrm{c}_{\mathrm{CdSe}}=5.2 \times 10^{-6} \mathrm{~mol}^{-1}$ (first plug), BGE $10 \mathrm{mM}$ sodium tetraborate, applied voltage $20 \mathrm{kV}$, detection UV-vis $245 \mathrm{~nm}$ (inset frame a, $\lambda=330 \mathrm{~nm}$ to monitor NCs only). Inset frame c, the two plugs system, without NCs (blank). Right side are graphic interpretations of each run, where upper is the starting situation and below is the situation observed at detector (orange, first plug; yellow, second plug and red, NCs). Note, that due to the NCs transport from the first plug to the plug/BGE boundary, both plugs $(1+2)$ are TX-100/DOSS layers free from NCs, denoted as yellow

of plug and related with this migration of NCs, (ii) compression of the length of the plug, depicted by sloping, dashed lines (Fig. S10). Compression of the plug, due to decrease in the content of an ionic amphiphile in a micellar segment in between BGE, is in agreement with ref. [43], and (iii) gradual broadening of NCs peak throughout the second plug (Fig. S10, frames e, d). Note, that this example shows the complexity in $\mathrm{NC} /$ plug system. Thus, taking into account conductivity of sample plug $(0.21 \mathrm{mS})$ vs. micellar plug $(0.011 \mathrm{mS})$ (plug 1 and 2, respectively; frame e, Fig. S10), as

well as difference in mobility, that sample plug $>$ micellar plug (frames d, e) as concluded from relation: mobility of mixed micelle $=\mathrm{f}$ (ratio non-ionic/ionic surfactant) from ref. [43], it can be stated that isotachophoretic version of electrophoresis can occur in this situation (remark of anonymous reviewer). On the other hand, only a plug with sufficient content of an ionic amphiphile can effectively support pseudomicellar state of NCs within plug. Evidently, insufficient DOSS content in a plug enforces a pseudomicelle to release surface bound amphiphiles to the environment, 
thereby precluding efficient accumulation of NCs at plug/ BGE boundary. Thus, the broadening of a peak of NCs observed here, can be due to one dominant effect or as a result of superposition of both effects mentioned.

Moreover, the effect of the peak broadening does prove again the presence of NCs being in the form of dispersed solutes rather than aggregates, since a shift in a peak shape, from focused peak (at high DOSS content) to broaden peak (at low DOSS content), concerns the state of a solute and should not impact the state of an aggregate.

The third and final experiment (Fig. 6) shows the effect of the length of second plug (range: $3.3-12 \mathrm{~cm}$ ) at constant length of a sample plug $(3.3 \mathrm{~cm})$. It was observed that despite ratio in lengths, up to four, the transport of NCs (first plug $\rightarrow$ second plug/BGE boundary) was found to be efficient since peak height of NCs was seen roughly independent from the length of the second plug. Obviously, this is confirmation of a pseudomicellar state of NCs as only this state (vs. aggregates) allows NCs to migrate efficiently in a micellar environment. On the other hand, the experiment (Fig. 6) shows a new, interesting situation that NCs from a sample plug (matrix) can be selectively transported into a pure micellar environment, far away from a matrix. This is a new method for solutes extraction from a sample, in combination with efficient preconcentration in the form of focused nanostructures and will be analysed in details in future works.

Effect of modification of electrolyte buffer on migration of $\mathrm{CdSe} / \mathrm{TOP} / /$ amphiphile $\mathrm{NCs}$

It was found that NCs could be forced to migrate outside the micellar plug by modifying electrolyte buffer-due to an increase in ionic strength or by adding organic modifiers, such as methanol or acetonitrile, to a background electrolyte buffer. This issue was shortly scrutinized in Fig. S9 (supporting materials) based on mentioned previous works. One of consequences of BGE modification is the separation of plain nanoparticles from these bioconjugated with DNA, which enables estimate yield of bioconjugation [19]. In the present work, conditions for NCs release from the plug was analyzed taking into account the conductivity factor for BGE and plug, respectively. For this BGE based on potassium chloride was used as this salt is used typically to conductometric experiments (e.g. calibration of conductivity electrode). Figure, which depicts this experiment, was posted on Electronic Support Materials, due to many details included (Fig. S11, supporting materials). In agreement with the previous report [19] and Fig. S9 (supporting materials), the NCs release from the plug to BGE was seen at higher ionic strength of BGE, that conductivity $\mathrm{BGE}>$ plug (frame f, Fig. S11). The related phenomenon in this context is an impact of BGE conductivity on the length of plug (denoted as $p=\mathrm{t}_{\mathrm{END}}-\mathrm{t}_{\mathrm{EOF}}$ ) (frame e, Fig. S11). It was seen that BGE ionic strength influences the $t_{\mathrm{END}}$ (border: plug end/BGE) more than $t_{\mathrm{EOF}}$ (beginning of the plug; side: $\mathrm{BGE} / \mathrm{plug}$ ), which is a sign of different composition of the plug front vs. the plug end. Note that, at $t=0$ a plug was homogeneous and at $\left(\mathrm{t}_{\text {run }}>0\right)$ the difference between plug front vs. end, in terms of composition, is due to electrophoresis. Note, that length of the plug $(p)$ can be regulated by the plug composition, that decrease in the content of an ionic amphiphile in a plug leads to decrease in a plug length (Fig. S10, supporting materials). The next factor - the peak area of the focused peak, monitored at $\lambda=330 \mathrm{~nm}$ (frames a-c, Fig. $\mathrm{S} 11$ ), shows that the parameter is insensitive to changes in plug length $(p)$. This leads to the conclusion that the border plug end/BGE can accommodate only a limited amount of NCs and additional increase beyond this limit leads to NCs peak broadening at the plug/BGE border or dispersion of NCs throughout the plug.

It was observed in the previous work that organic solvents added to the plug can effectively release NCs from the plug [19]. Based on conductivity experiment, some salts $(\mathrm{Li}, \mathrm{Na}, \mathrm{K}$, all $\mathrm{Cl}$ ) were checked and additives, typically $\mathrm{MeOH}$ or $\mathrm{ACN}$, lead to decrease or increase the conductivity of an appropriate salt solution, respectively. However applying as BGE the sodium tetraborate containing $\mathrm{ACN}$, the conductivity was found to be fairly constant despite an increase in ACN content in BGE (Fig. 7, frame d). In this situation, NCs exit the plug (Fig. 7, frames b, c) is the effect of the presence of $\mathrm{ACN}$ in BGE (conductivity $_{\mathrm{BGE}}=$ constant). On the other hand, under the reverse situation, that ACN was added before a run to the sample plug instead of BGE, even higher content of ACN in a sample plug did not lead to NCs release from a plug (Fig. 7, frame d), which definitely confirms, controllable by a BGE, a release of NCs from a plug/BGE boundary.

There are two final remarks related with issues discussed here. First one concerns a peak shape of NCs being released from a plug, that in some cases the peak is sharp and in some broaden. This issue is shortly discussed in text that accompanies frame g, Fig S11 (supporting materials), as well as in the last section of the present work. Finally, separation of two NCs of different dimensions, using BGE modified by $\mathrm{ACN}$ is discussed (Fig. S12, supporting materials) with the conclusion that although an efficient separation of two CdSe $\mathrm{NC}$ was obtained, the reason for this is difference in the state of surface of crystal CdSe.

Simplified mechanism for focusing and release of CdSe/amphiphile NCs using a micellar plug

It can be stated, based on capillary electrophoresis experiments, that at least three effects leads to focusing of $\mathrm{CdSe} /$ 
Fig. 7 Effect of modification of BGE or plug by acetonitrile $(\mathrm{ACN})$. A sample used as a plug was CdSe NCs dispersed in TX100/sodium laurate (LA) (10\% w:w/0.13 M). Frames: a, BGE $10 \mathrm{mM}$ sodium tetraborate; $\mathbf{b}$, BGE $10 \mathrm{mM}$ sodium tetraborate containing 15\% ACN; c, BGE $10 \mathrm{mM}$ sodium tetraborate containing $30 \%$ ACN. Frame d, modification of a plug by $\mathrm{ACN}$ (1:1); BGE, $10 \mathrm{mM}$ sodium tetraborate. Peaks: $\mathrm{NC}_{\text {plug }}$, CdSe $\mathrm{NCs}$ being in a plug; $\mathrm{NC}_{\mathrm{BGE}}$, CdSe NCs released from a plug to BGE. Conditions: voltage $20 \mathrm{kV}$, detection UV-vis $330 \mathrm{~nm}$ in order to visualize NCs only, injection $50 \mathrm{mbar} / 0.1 \mathrm{~min}$. Each frame was set at the same absorbance scale in order to visualize the effect
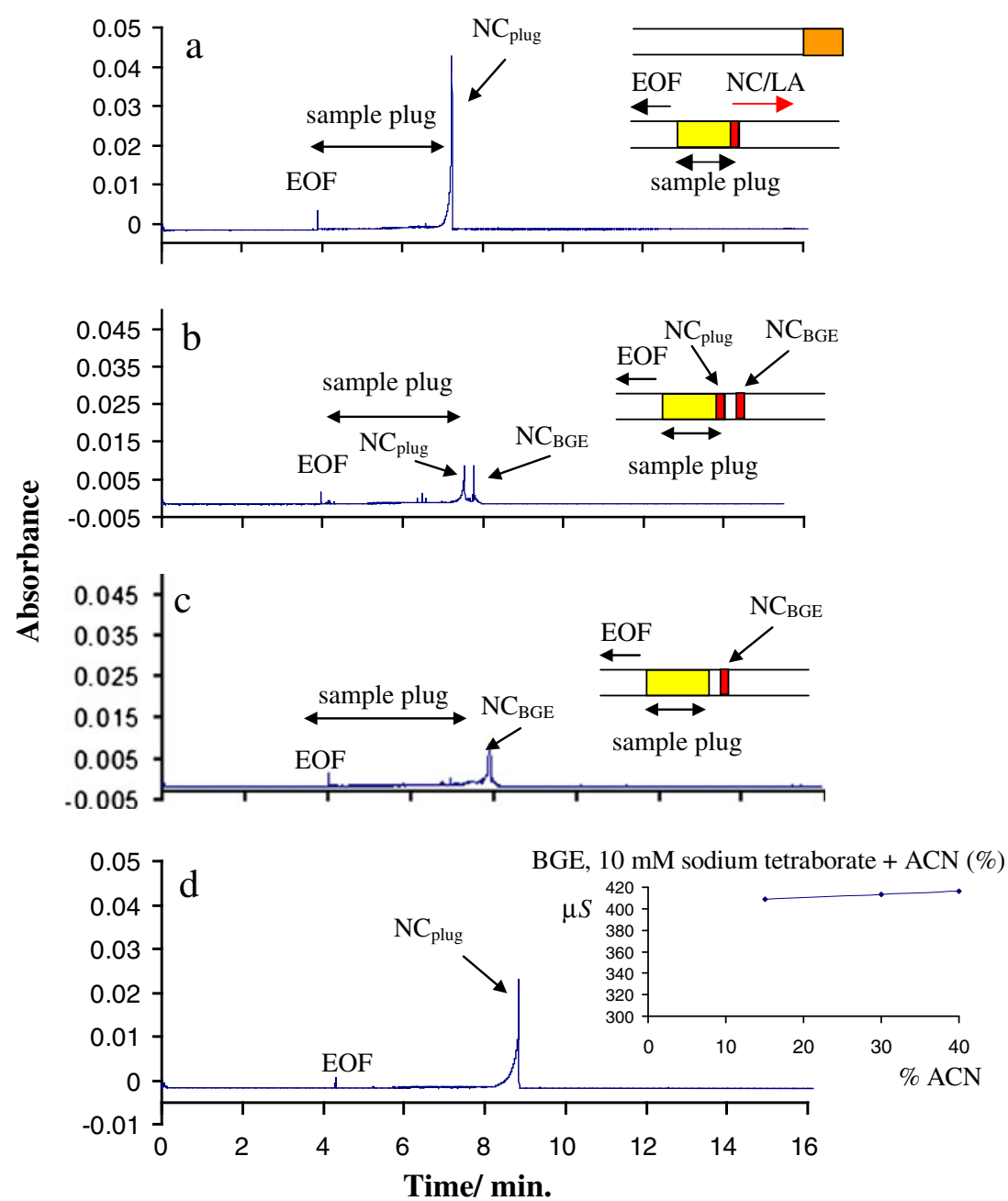

amphiphile NCs using a plug of mixed micelles. These are: the presence of a plug - thus a segment of phase of mixed micelles in between BGE, high affinity of these NCs to a phase of micelles and additional coating of these NCs with amphiphiles during a run. On the other hand, the migration phenomena observed in the present work may be a special case of mixed micellar system, already analyzed by capillary electrophoresis [45, 46]. This is because CdSe/amphiphile NCs can be regarded as micelle-like objects able to form stable phase with regular micelles as concluded from the first part of the present work and refs. [18-20]. It was reported, that peaks of various fractions of mixed micelles with mobility between 0 and $-3.5 \times 10^{-4} \mathrm{~cm}^{2} \mathrm{~V}^{-1} \mathrm{~s}^{-1}$ were observed, depending on polydispersity in terms of size and composition of mixed micelles $[45,46]$. The same can be concluded from ref. [43], that specifically for mixed micelles, mobility depends on ratio: non-ionic/ionic surfactant in a phase of mixed micelles. The mobility is reduced in parallel with a decrease in concentration of an ionic surfactant in a phase of mixed micelles and for the fraction of an ionic surfactant in the phase: $1,04,0.3$ and 0.2 , the mobility: $-3.16,-2.55,-2.15$ and $-1.67\left(\times 10^{-4} \mathrm{~cm}^{2} \mathrm{~V}^{-1} \mathrm{~s}^{-1}\right)$ was estimated, respectively [43].

Focusing of $\mathrm{CdSe} / \mathrm{amphiphile} \mathrm{NCs} \mathrm{can} \mathrm{be} \mathrm{explained}$ based in this remark and the fact reported in the first part of the present work, that coating of CdSe NCs with a pair of amphiphiles is selective, that CdSe nanocrystal coated with an ionic amphiphile is the main product obtained (Fig. 2 and S7, Electronic Support Section). Thus, the whole system can be described in terms of electrically charged NCs dispersed in a phase of mixed micelles. In this situation, there is substantial difference in mobility: CdSe/ (ionic amphiphile) vs. mixed micelle, that mobility of $\mathrm{CdSe} /($ ionic amphiphile) $>>$ mobility of mixed micelle, in agreement with the relation above. The diversity in mobility $\mathrm{CdSe} /($ ionic amphiphile) vs. mixed micelle in a micellar plug is enhanced due to the substantial reduction of the drag force for a particle migrated in a micellar phase, because of very low viscosity of the micellar medium.

In order to discus a release of $\mathrm{CdSe} / \mathrm{amphiphile} \mathrm{NCs}$ from a micellar plug it should be noted that a lack of broadening of a peak of focused NCs in a plug, even 
despite long run (ca. 50 min, Fig. 4), means that BGE has no access to plug/BGE boundary containing CdSe/amphiphile NCs. This confirms the state of a micellar plug as a separate phase migrated in between BGE, which does not exchange its contents with BGE. A release of $\mathrm{CdSe} /$ amphiphile NCs from a plug is the consequence of the modification of BGE. In this case, a decrease in integrity of a micellar system at plug/BGE boundary, does lead to a release of CdSe/amphiphile NCs from a plug to BGE. Depending on the type of BGE modification (organic solvents, ionic strength) and state of a nanocrystal (e.g. size), released from a plug, the CdSe/amphiphile can form either sharp $\left(N \sim 10^{7}\right)$ or broaden $\left(N \sim 10^{5}\right)$ peak in BGE (example see frame g, Fig. S11, Electronic Support Section). Obviously, in this instance, a lack of support from relatively high concentration of amphiphiles in a phase of mixed micelles, enables exit of an ionic amphiphile, attached to surface of a nanocrystal, to aqueous phase of BGE. This results in a decrease in charge density of a particle, that leads to a decrease in $\zeta$-potential and if $\zeta$-potential falls below a threshold value, according to DLVO theory a coagulation of particles takes place. This effect influences the present state of the CdSe/amphiphile particle in BGE, that balance between nanocrystals dispersed vs. coagulated in BGE ultimately impacts the peak shape of these NCs in BGE.

\section{Conclusions}

In the present work, the hydrophobic CdSe/TOP NCs were functionalized using amphiphilic molecules. Two types of NCs coating with amphiphiles have been distinguished, due to the difference in coating mechanisms, regulated by the specific affinity of ligands to a surface of the CdSe crystal. This issue was discussed in the light of data obtained from molecular modeling at DFT level, applied to estimate important interactions in $\mathrm{CdSe} / \mathrm{amphiphile} \mathrm{systems.} \mathrm{Analy-}$ sis of electrophoretic migrations for $\mathrm{CdSe} / \mathrm{TOP} / /$ amphiphile NCs leads to the conclusion that these solutes can be regarded as pseudomicelles, able to form a mixed pseudomicellar system with regular micelles. Because of this, using a micellar plug, the CdSe/TOP//amphiphile NCs can be focused at the boundary between micellar and micelle-free zone. Finally, conditions for demixing of the mixed pseudomicellar system, which allows NCs to be released from the micellar plug, were analyzed with the conclusion that the effect can be controlled by the current electrophoretic conditions.

Acknowledgements - the U.S. Air Force Research Laboratory and Air Force Office of Scientific Research — grant number FA9550-06-1-0365,

- Ministry of Science and Higher Education (Poland) - grant number N 204142837.
Open Access This article is distributed under the terms of the Creative Commons Attribution Noncommercial License which permits any noncommercial use, distribution, and reproduction in any medium, provided the original author(s) and source are credited.

\section{References}

1. Somers RC, Bawendi MG, Nocera DG (2007) CdSe nanocrystal based chem-/bio- sensors. Chem Soc Rev 36:579-591

2. Yu WW, Qu L, Guo W, Peng X (2003) Experimental determination of the extinction coefficient of $\mathrm{CdTe}, \mathrm{CdSe}$, and $\mathrm{CdS}$ nanocrystals. Chem Mater 15:2854-2860

3. Sergeev GB (2006) Nanochemistry. Elsevier B.V, Amsterdam

4. Pons T, Uyeda HT, Medintz IL, Mattoussi H (2006) Hydrodynamic dimensions, electrophoretic mobility, and stability of hydrophilic quantum dots. J Phys Chem B 110:20308-20316

5. Radko SP, Chrambach A (2002) Separation and characterization of sub- $\mu \mathrm{m}$ - and $\mu \mathrm{m}$-sized particles by capillary zone electrophoresis. Electrophoresis 23:1957-1972

6. Rodriguez MA, Armstrong DW (2004) Separation and analysis of colloidal/nano-particles including microorganisms by capillary electrophoresis: a fundamental review. J Chromatogr B 800:7-25

7. Liu F-K (2009) Analysis and applications of nanoparticles in the separation sciences. A case of gold nanoparticles. J Chromatogr A 1216:9034-9047

8. Surugau N, Urban PL (2009) Electrophoretic methods for separation of nanoparticles. J Sep Sci 32:1889-1906

9. López-Lorente AI, Simonet BM, Valcárcel M (2011) Electrophoretic methods for the analysis of nanoparticles. Trends Anal Chem 30:58-71

10. Pyell U (2008) CE characterization of semiconductor nanocrystals encapsulated with amorphous silicium dioxide. Electrophoresis 29:576-589

11. Delgado AV, González-Caballero F, Hunter RJ, Koopal LK, Lyklema J (2007) Measurement and interpretation of electrokinetic phenomena. J Colloid Interface Sci 309:194-224

12. Song X, Li L, Qian H, Fang N, Ren J (2006) Highly efficient size separation of CdTe quantum dots by capillary gel electrophoresis using polymer solution as sieving medium. Electrophoresis 27:1341-1346

13. Li Y-Q, Wang H-Q, Wang J-H, Guan L-Y, Liu B-F, Zhao Y-D, Chen H (2009) A highly efficient capillary electrophoresis-based method for size determination of water-soluble CdSe/ZnS coreshell quantum dots. Anal Chim Acta 647:219-225

14. Lin K-H, Chu T-C, Liu F-K (2007) On-line enhancement and separation of nanoparticles using capillary electrophoresis. J Chromatogr A 1161:314-321

15. Liu F-K (2007) A high-efficiency capillary electrophoresis-based method for characterizing the sizes of $\mathrm{Au}$ nanoparticles. J Chromatogr A 1167:231-235

16. Liu F-K (2009) Using micellar electrokinetic chromatography for the highly efficient preconcentration and separation of gold nanoparticles. J Chromatogr A 1216:2554-2559

17. Pyell U, Bücking W, Huhn C, Herrmann B, Merkoulov A, Mannhardt J, Jungclas H, Nann T (2009) Calibration-free concentration determination of charged colloidal nanoparticles and determination of effective charges by capillary isotachophoresis. Anal Bioanal Chem 395:1681-1691

18. Oszwałdowski S, Zawistowska K, Grisby L, Roberts KP (2010) Capillary electrophoretic separation and characterizations of CdSe quantum dots. Cent Eur J Chem 8:806-819

19. Oszwałdowski S, Zawistowska-Gibuła K, Roberts KP (2011) Capillary electrophoretic separation of nanoparticles. Anal Bioanal Chem 399:2831-2842 
20. Oszwałdowski S, Zawistowska-Gibuła K, Roberts KP (2011) Characterization of CdSe quantum dots with bidentate ligands by capillary electrophoresis. Cent Eur J Chem 9:572-584

21. Patra HK, Sarkar DG, Dasgupta AK (2009) Multimodal electrophoresis of gold nanoparticles: A real time approach. Anal Chim Acta 649:128-134

22. Dubertret B, Skourides P, Norris D, Noireaux V, Brivanlou AH, Libchaber (2002) A In vivo imaging of quantum dots encapsulated in phospholipid micelles. Science 298:1759-1762

23. Fan $\mathrm{H}$, Leve EW, Scullin Ch, Gabaldon J, Tallant D, Bunge S, Boyle T, Wilson MC, Brinker CJ (2005) Surfactant-assisted synthesis of water-soluble and biocompatible semiconductor quantum dot micelles. Nano Lett 5:645-648

24. Zhelev Z, Ohba H, Bakalova R (2006) Single quantum dotmicelles coated with silica shell as potentially non-cytotoxic fluorescent cell tracers. J Am Chem Soc 128:6324-6325

25. Bakalova R, Zhelev Z, Aoki I, Ohba H, Imai Y, Kanno I (2006) Silica-shelled single quantum dot micelles as imaging probes with dual or multimodality. Anal Chem 78:5925-5932

26. Jin T, Fujii F, Yamada E, Nodasaka Y, Kinjo M (2006) Control of the optical properties of quantum dots by surface coating with calix[n]arene carboxylic acids. J Am Chem Soc 128:9288-9289

27. Islam MF, Rojas E, Bergey DM, Johnson AT, Yodh AG (2003) High weight fraction surfactant solubilization of single-wall carbon nanotubes in water. Nano Lett 3:269-273ANO

28. Moore VC, Strano MS, Haroz EH, Hauge RH, Smalley RE, Schmidt J, Talman Y (2003) Individually suspended single-walled carbon nanotubes in various surfactants. Nano Lett 3:1379-1382

29. Wenseleers W, Vlasov II, Goovaerts E, Obraztsova ED, Lobach AS, Bouwen A (2004) Efficient isolation and solubilization of pristine single-walled nanotubes in bile salt micelles. Adv Funct Mater 14:1105-1112

30. Treubig JM, Brown PR (2000) Novel approach to the analysis and use of fullerenes in capillary electrophoresis. J Chromatogr A 873:257-267

31. Arnold MS, Green AA, Hulvat JF, Stupp SI, Hersam MC (2006) Sorting carbon nanotubes by electronic structure using density differentiation. Nat Nanotechnol 1:60-65

32. Doorn SK, Fields RE, Hu H, Hamon MA, Haddon RC, Selegue JP, Majidi V (2002) High resolution capillary electrophoresis of carbon nanotubes. J Am Chem Soc 124:3169-3174

33. Oszwałdowski S, Vasicek L, Tonev A, Roberts KP (2007) Characterization of CdSe nanocrystals and their interaction with micellar aggregates by means of capillary electrophoretic techniques. Nanotech Conference Technical Proceedings 4:332335

34. Bulavchenko AI, Pletnev DN (2008) Electrophoretic concentration of nanoparticles of gold in reversed micellar solutions of AOT. J Phys Chem C 112:16365-16369

35. Bulavchenko AI, Popovetsky PS (2010) Electrokinetic potential of nanoparticles in reverse AOT micelles: photometric determination and role in the processes of heterocoagulation, separation, and concentration. Langmuir 26:736-742

36. Asokan S, Krueger KM, Alkhawaldeh A, Carreon AR, Mu Z, Colvin VL, Mantzaris NV, Wong MS (2005) The use of heat transfer fluids in the synthesis of high-quality CdSe quantum dots, core/shell quantum dots, and quantum rods. Nanotechnology 16:2000-2011

37. Landers JP (1997) Handbook of capillary electrophoresis. CRC Press, Danvers (page 870)

38. NN-LABS, www.nn-labs.com/cdsesizingcurve.html

39. NN-LABS, www.nn-labs.com/cdseeccurve.html

40. Moreels I, Martins JC, Hens Z (2006) Ligand adsorption/ desorption on sterically stabilized InP colloidal nanocrystals: observation and thermodynamic analysis. ChemPhysChem 7:1028-1031

41. Pramauro E, Pelizzetti E (1996) Surfactants in Analytical Chemistry. Wilson \& Wilson's, Elsevier, Amsterdam

42. Han R, Yu M, Zheng Q, Wang L, Hong Y, Sha Y (2009) A facile synthesis of small-sized, highly photoluminescent, and monodisperse CdSeS QD/SiO 2 for live cell imaging. Langmuir 25:1225012255

43. Asakawa T, Sunazaki S, Miyagishi S (1992) Electromigration of ionic micelles by use of an isotachophoretic apparatus. Colloid Polym Sci 270:259-266

44. Liu F-K, Wei G-T (2004) Adding sodium dodecylsulfate to the running electrolyte enhances the separation of gold nanoparticles by capillary electrophoresis. Anal Chim Acta 510:77-83

45. Zhang B, Kirton GF, Dubin PL (2002) Compositional heterogeneity in anionic/nonionic mixed micelles observed by frontal analysis continuous capillary electrophoresis. Langmuir 18:46054609

46. Zhang H, Dubin PL (1997) Analysis of polydispersity of mixed micelles of TX-100/SDS and $\mathrm{C}_{12} \mathrm{E}_{8} / \mathrm{SDS}$ by capillary electrophoresis. J Colloid Interface Sci 186:264-270 